EPJ Web of Conferences 45, 01060 (2013)

DOI: $10.1051 /$ epjconf/20134501060

C) Owned by the authors, published by EDP Sciences, 2013

\title{
Application of Positron Emission Tomography to Aerosol Transport Research in a Model of Human Lungs
}

\author{
F. Lizal ${ }^{1}$, J. Jedelsky ${ }^{1}$, J. Adam ${ }^{2,3}$, M. Belka ${ }^{1}$, and M. Jicha ${ }^{1}$ \\ ${ }^{1}$ Brno University of Technology, Faculty of Mechanical Engineering, Energy Institute, Technicka 2896/2, 61669 Brno, \\ Czech Republic \\ ${ }^{2}$ UJVRez a.s., Division of Radiopharmaceuticals, Hlavni 130, 250 68,Husinec-Rez, Czech Republic \\ ${ }^{3}$ Masaryk Memorial Cancer Institute, Zluty kopec 7a, 65653 Brno
}

\begin{abstract}
Positron Emission Tomography (PET) is a convenient method for measurement of aerosol deposition in complex models of lungs. It allows not only the evaluation of regional deposition characteristics but also precisely detects deposition hot spots. The method is based on a detection of a pair of annihilation photons moving in opposite directions as a result of positron - electron interaction after the positron emission decay of a suitable radioisotope. Liquid di(2-ethylhexyl) sebacate (DEHS) particles tagged with fluorine-18 as a radioactive tracer were generated by condensation monodisperse aerosol generator. Aerosol deposition was measured for three different inhalation flowrates and for two sizes of particles. Combination of PET with Computed Tomography (CT) in one device allowed precise localisation of particular segments of the model. The results proved correlation of deposition efficiency with Stokes number, which means that the main deposition mechanism is inertial impaction. As a next task the methodology for tagging the solid aerosol particles with radioactive tracer will be developed and deposition of porous and fiber aerosols will be measured.
\end{abstract}

\section{Introduction}

Inhaled aerosolized medication has a great potential to contribute to more effective, comfortable and cheaper treatment of both lung and systemic diseases. The key factor is to deliver the aerosol to the intended region of lungs. For successful targeted delivery of aerosol is necessary to identify parameters affecting transport and deposition of aerosols in human respiratory tract. General description of mechanisms controlling deposition of aerosol in human lungs was published by Lippmann [1] Swift [2] and Heyder [3]. Particle may deposit by the effect of following deposition mechanisms:

1) interception - when particle following its natural gas streamline contacts the surface of lungs due to its physical size

2) inertial impaction - when air changes its direction, high inertia particles are unable to follow the streamlines, they continue in straight direction and deposit on the lung surface

3) sedimentation - settling of particles due to gravitational forces in smaller airways with low air velocities

4) diffusion - predominant deposition mechanism for submicrometer particles
5) electrostatic precipitation - highly charged particles can induce image charge on the lung surface and hence be attracted to the surface.

In most cases the inertial impaction, sedimentation and diffusion are dominant deposition mechanisms [1]. The influence of inertial forces on particle depends on its aerodynamic diameter rather than on its physical size. Aerodynamic diameter is an equivalent diameter defined as the diameter of the spherical particle with a density of $1000 \mathrm{~kg} / \mathrm{m}^{3}$ that has the same settling velocity as the particle [4]. The aerosol deposition induced by inertial impaction is usually expressed in terms of Stokes number, which is defined as:

$$
\text { Stk }=\frac{\rho d_{p}^{2} U}{18 \mu D}
$$

where $\rho\left(\mathrm{kg} / \mathrm{m}^{3}\right)$ is the particle density, $d_{p}(\mathrm{~m})$ is the aerodynamic diameter of the particle, $U(\mathrm{~m} / \mathrm{s})$ is the flow velocity, $\mu(\mathrm{Pa} \cdot \mathrm{s})$ is the air viscosity and $D(\mathrm{~m})$ is a characteristic dimension, in our case a diameter of the airway.

Particles larger than $1 \mu \mathrm{m}$ deposit mainly due to inertial impaction in large airways. Sedimentation is most important in smaller airways and in alveolar region and it has maximal effect in horizontally oriented airways [4]. 
Diffusion takes place in whole respiratory tract, but is more noticeable in smaller airways where distances are shorter and the residence time is longer.

Targeted delivery of aerosolized medication is considered successful if at least $50 \%$ of inhaled aerosol deposit in desired region [5]. Transport of the medication to large and/or small conducting airways represent the most complicated issue. At the same time medication for treatment of asthma, chronic obstructive pulmonary disease, or cystic fibrosis are required to be delivered there. Majority of currently marketed devices provide particles in range of 1 to $5 \mu \mathrm{m}$, with the understanding that there may be considerable oropharyngeal and alveolar surface exposure to the drug, which could be sufficient for some inhaled agents; nevertheless, for those drugs that are extremely costly, or have harmful side effects, an improved delivery is needed. Current devices reach only the level of $30 \%$ deposition of inhaled aerosol in conducting airways [5,6].

The effort to develop devices capable of delivering the medication to conductive airways with higher efficiency is conditioned by deeper understanding to mechanisms of transport and deposition of aerosol. Therefore in vitro experiments are performed with various sizes and types of particles for different breathing regimes. In this paper we present results of regional aerosol deposition measured on realistic segmented model of lungs using Positron Emission Tomography (PET).

\section{Methods}

Measurement was performed on the realistic transparent model of human lungs published in [7]. The model consists of 32 segments and has 10 outputs. It comprises respiratory airways from oral cavity to the seventh generation of branching (please see figure 1).

PET is a commonly used method in nuclear medicine. The method is based on a detection of a pair of simultaneously emitted annihilation photons moving in opposite directions as a result of positron - electron interaction after the positron emission decay of a suitable radioisotope. For the purpose of PET examination, the most commonly used radiopharmaceutical is Fluorodeoxyglucose (FDG) with the positron-emitting isotope fluorine-18 substituted for the one of the hydroxyl groups in the glucose molecule. In the beginning of medical examination FDG is injected to the patient. Tissues with the highest metabolic activity (typically tissues with tumor activity) have the highest FDG uptake and hence absorb higher quantity of FDG. The organs stricken by cancer can therefore be identified from the PET images due to increased activity detected by the PET scanner.

PET is also a convenient method for measurement of aerosol deposition in complex models of lungs. In our case the liquid aerosol particles from di(2-ethylhexyl) sebacate (DEHS) were generated by condensation monodisperse aerosol generator (CMAG) and labeled with fluorine-18 as a radioactive tracer. PET allows not only for the evaluation of regional deposition characteristics but also precisely detects deposition hot spots.

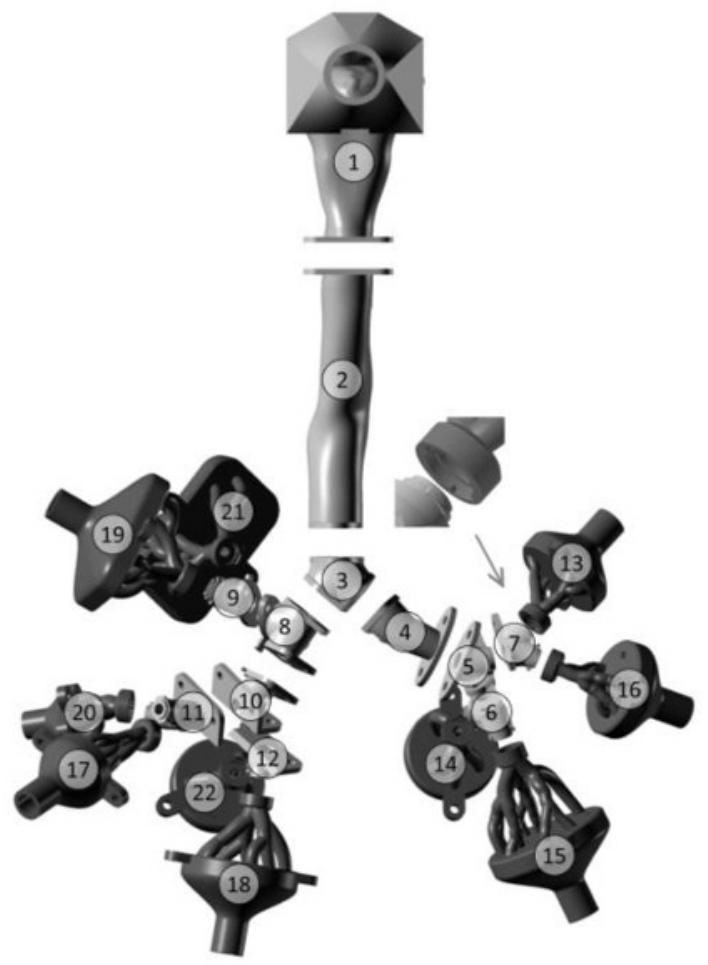

Fig. 1. The realistic segmented model of lungs.

A scheme of the experimental setup is presented in figure 2. CMAG was placed in shielded lead box to prevent radiation exposure of the laboratory personnel. Monodisperse aerosol was led through the ${ }^{85} \mathrm{Kr}$-based charge neutralizer to Process Aerosol Monitor (PAM), which measured size and concentration of aerosol. Dilutor served for mixing of the concentrated aerosol flow with air to achieve the desired inhalation flowrate. Three different inhalation flowrates $(15,30$ and $60 \mathrm{~L} / \mathrm{min}$ ) were used during this study.

Aerosol particles which did not deposit in the model were collected by output filters following each of the model outputs. Flowrates through the outputs were set according to realistic conditions. The model with output filters was transported to a hybrid PET/CT scanner (Siemens Biograph 64) right after the exposure. Resulting three-dimensional images were subsequently processed using software Carimas 2.4 [8] and total activities in each segment were calculated. Combination of PET with Computed Tomography (CT) in one device allowed precise localisation of particular segments of the model.

Aerosol deposition was measured for two sizes of particles $(2.5 \mu \mathrm{m}$ and $4.3 \mu \mathrm{m})$ to cover maximal range of Stokes numbers. Deposition characteristics were calculated from the activities. Deposition fraction is a ratio of the number of particles deposited in a given segment to the number of particles entering the model. Deposition efficiency is a ratio of the number of particles deposited in a given segment to the number of particles entering the segment. 


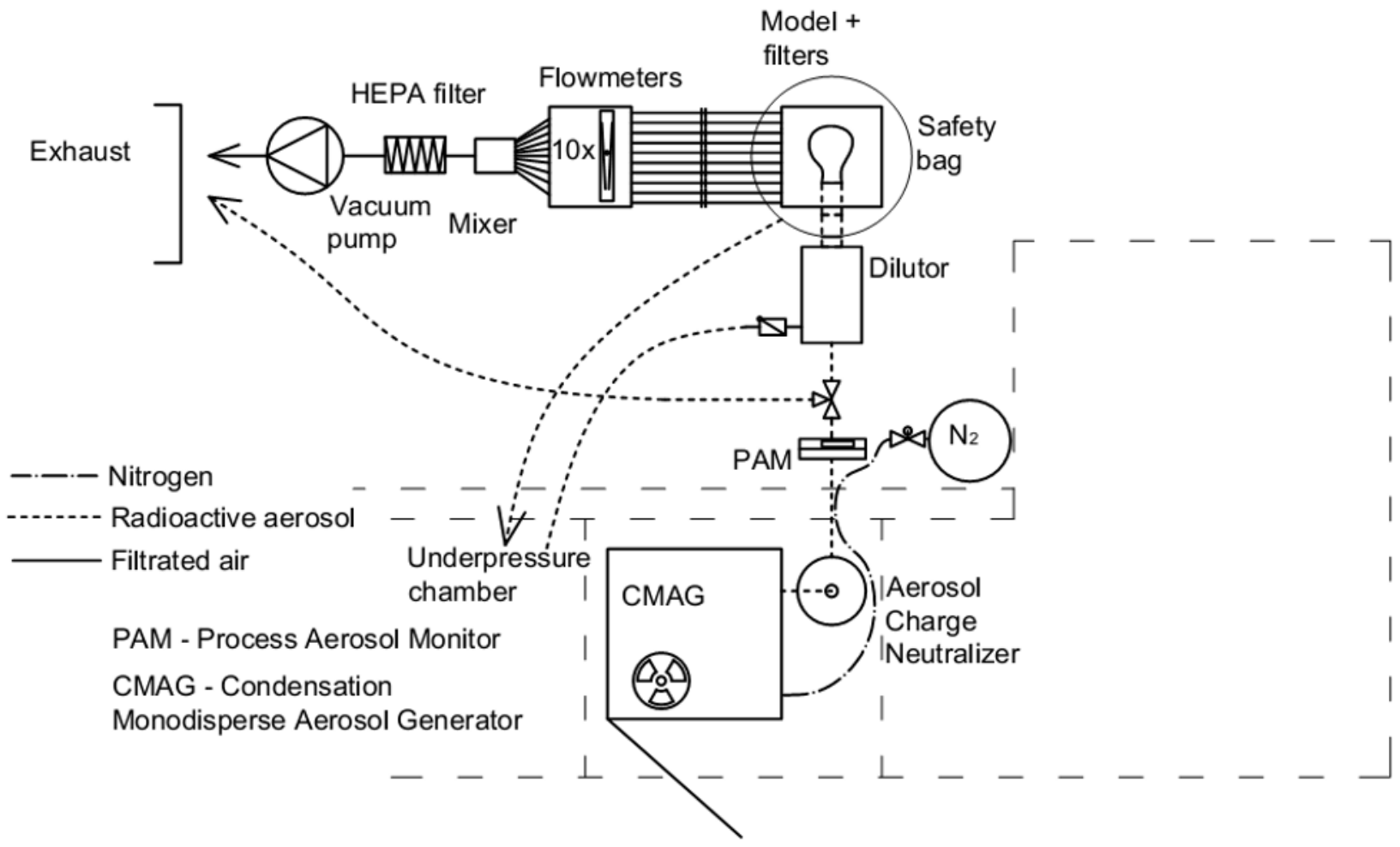

Fig. 2. A scheme of the experimental setup.

\section{Results and discussion}

Obvious increase of deposition fraction in segments 23 to 32 , which represent output filters, can be seen in the graph in figure 3. Distribution of deposition fraction on filters is identical for all measured regimes and corresponds to the distribution of flowrates through the outputs of the model. The difference of one order of magnitude can be seen among different regimes for segments 1 to 22 . The lowest deposition fraction was measured for particles in size of $2.5 \mu \mathrm{m}$ and flowrate $30 \mathrm{~L} / \mathrm{min}$. For all regimes the highest deposition fraction was found in segments 1 and 2 which represent oral cavity and trachea, respectively. The difference among other segments was almost negligible.

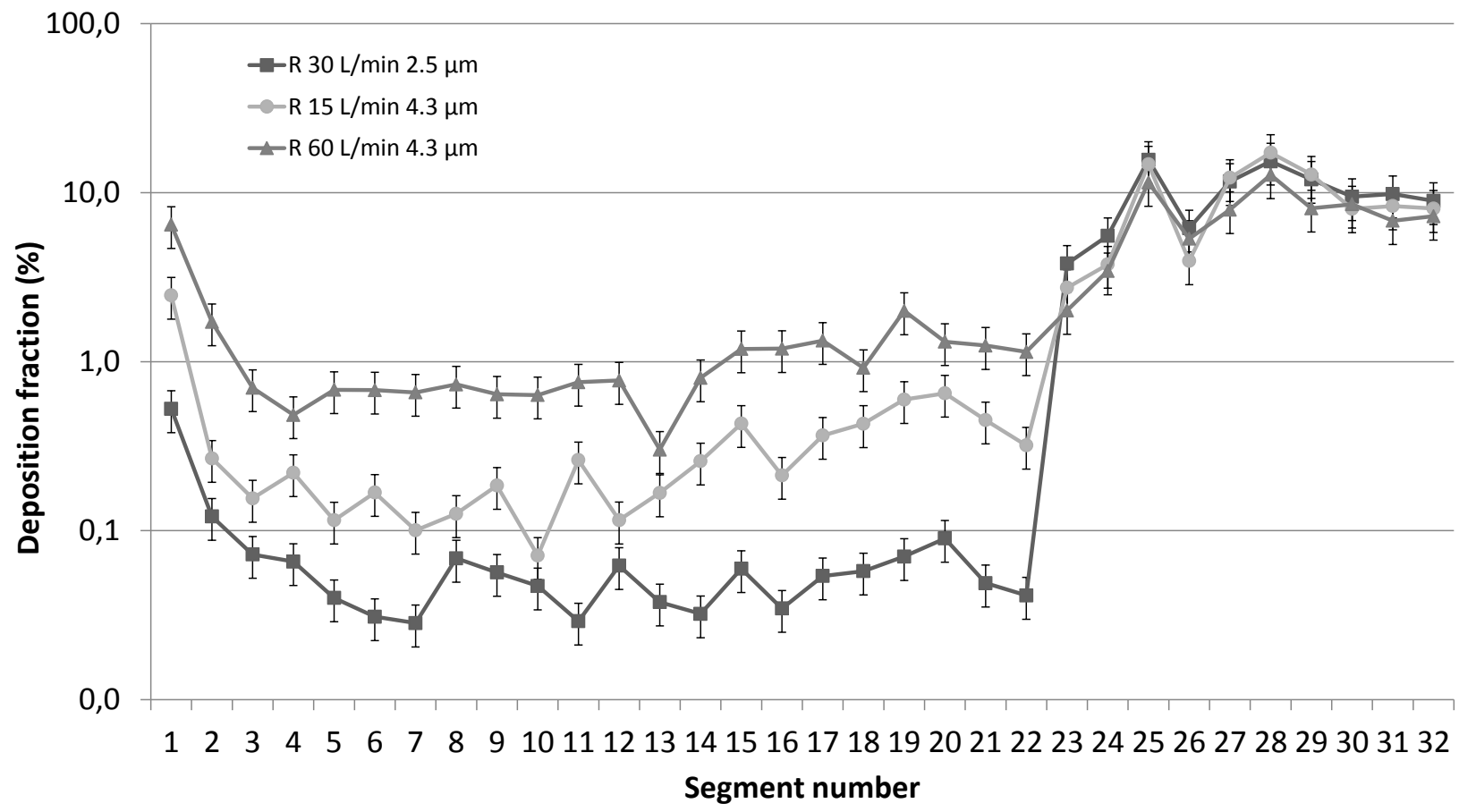

Fig. 3. Deposition fraction on the realistic model 


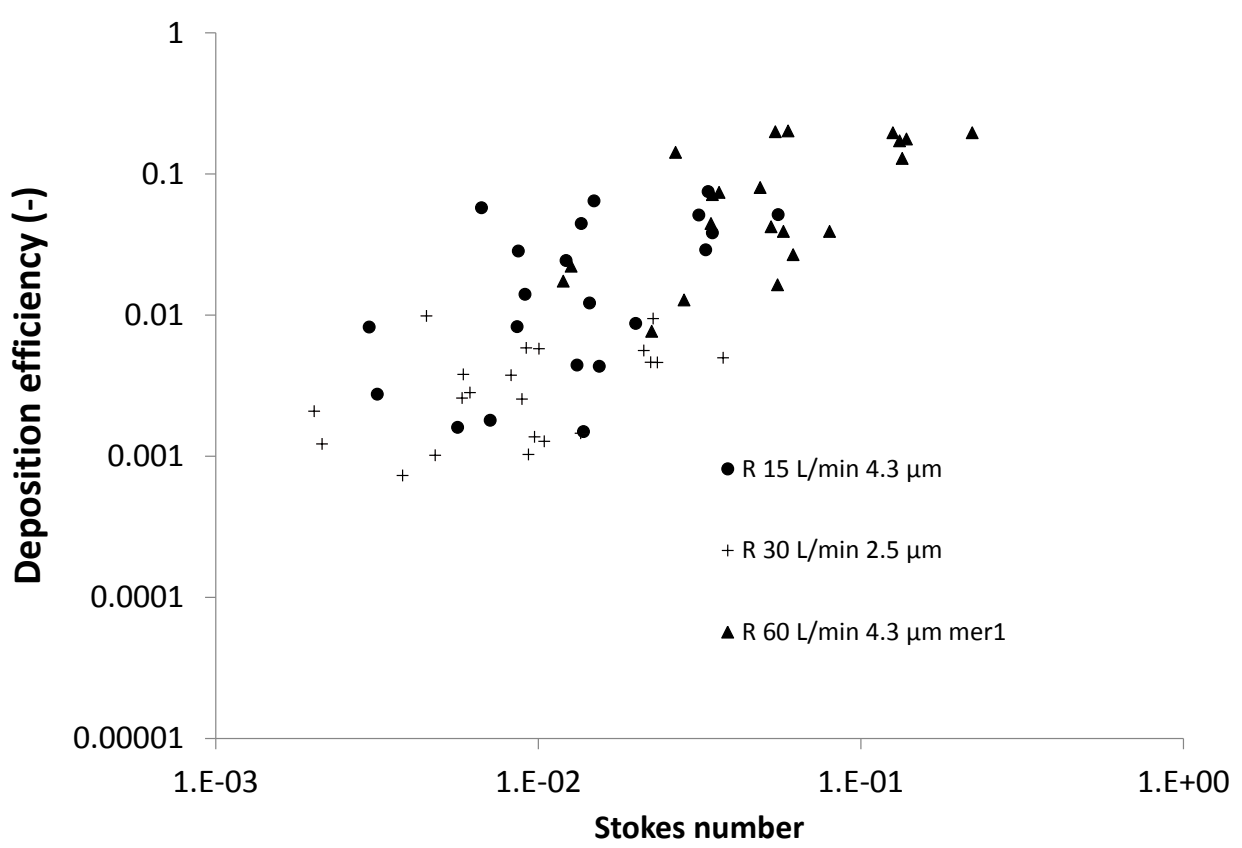

Fig. 4. Deposition efficiency on the realistic model

Only moderate increase can be seen for regimes with higher Stokes number (with $4.3 \mu \mathrm{m}$ particles). This fact could be surprising, as the segments 13 to 22 encompass multiple bifurcations which are known to be the deposition hot spots. Comparison of deposition in segments using deposition fraction is influenced by the fact that different amount of aerosol is entering different segments. For that reason deposition efficiency is preferred for inter-segment comparison.

Deposition efficiency in dependence on Stokes number is presented in figure 4. It is obvious that deposition efficiency increases with increasing Stokes number. Therefore inertial impaction is confirmed as a dominant deposition mechanism for particles in sizes of 2.5 and $4.3 \mu \mathrm{m}$ in airways out to $7^{\text {th }}$ generation of branching.

The results acquired for liquid spherical particles on the realistic model serve as a base for comparison with deposition of fibers and porous particles. Aerodynamic diameter is significantly different from physical size for both mentioned types of particles; therefore major differences are expected in deposition characteristics.

\section{Summary}

Measurement of spherical aerosol particles was performed on the realistic model of lungs using PET. Deposition characteristics were calculated based on the analysis of three dimensional PET images. Tagging of liquid monodisperse particles by radioactive tracer was solved using standard condensation monodisperse aerosol generator, where positron-emitting isotope fluorine-18 was added to the atomizer. As a next task the methodology for tagging solid aerosol particles with suitable radioactive tracer will be developed and deposition of porous and fiber aerosols will be measured.

\section{Acknowledgement}

The authors gratefully acknowledge the help of Tomas Dvoracek from UJVRez a.s., Division of Radiopharmaceuticals and Karol Bolcak from Masaryk Memorial Cancer Institute Brno. This work was supported by the Czech Grant Agency under the grant GA P105/11/1339, by the project ME 09030 of the program KONTAKT supported by the Czech Ministry of Education, Youth and Sports, by the project FSI-S-11-6, and by the European Regional Development Fund and the State Budget of the Czech Republic (RECAMO, CZ.1.05/2.1.00/03.0101).

\section{References}

1. M. Lippmann, D. B. Yeates, and R. E. Albert, British Journal of Industrial Medicine 37 (4), 337 (1980).

2. D. L. Swift, Annals of Biomedical Engineering 9 (5-6), 593 (1981).

3. J. Heyder, European Journal of Respiratory Diseases 63, 29 (1982).

4. W. C. Hinds, Aerosol technology : properties, behavior, and measurement of airborne particles, 2nd ed. (Wiley, New York, 1999).

5. J. Heyder, in Proc Am Thorac Soc (United States, 2004), Vol. 1, pp. 315.

6. K. L. Zeman, J. H. Wu, and W. D. Bennett, Journal of Aerosol Medicine and Pulmonary Drug Delivery 23 (6), 363 (2010).

7. F. Lizal, J. Elcner, P. K. Hopke, J. Jedelsky, and M. Jicha, Proc. IMechE Part H-Journal of Engineering in Medicine 226 (H3), 197 (2012).

8. Turku PET Centre, (2012). on-line [cited 26.3.2012]. Available from Internet: <http://www.turkupetcentre.fi/>. 